\title{
Efecto del fotoperiodo sobre la calidad comercial de plantas de Althaea rosea obtenidas a partir de germoplasma elite
}

Papone, M.L. y N. Fatta

\begin{abstract}
RESUMEN
Althaea rosea L. tiene escasa difusión en Buenos Aires a pesar de su valor decorativo. Partiendo de semillas de plantas asilvestradas y después de tres ciclos de selección, se logró germoplasma con buen comportamiento en macetas. Con la finalidad de difundir $A$. rosea, se encaró un ensayo en macetas cuyo objetivo fue estimar el efecto del fotoperíodo sobre la calidad comercial del germoplasma elite obtenido. Las plantas que recibieron $16 \mathrm{~h}$ de luz mostraron menor número de días hasta la aparición del primer pimpollo que aquellas que recibieron luz natural. Este resultado es interesante, pues indica que las primeras podrían llegar anticipadamente a la venta. También se hallaron diferencias para área y longitud de pétalo a favor de las plantas con $16 \mathrm{~h}$ de fotoperíodo, las que mostraron flores de mayor tamaño y pétalos más anchos que tendieron a solaparse al compararlas con lo observado en las flores de plantas que recibieron luz natural. Las plantas tratadas con fotoperíodo largo exhibieron menores coeficientes de variación para los caracteres medidos, lo que permite concluir que la suplementación con luz es una herramienta efectiva para concentrar la operación de venta y para aumentar la calidad de plantas de A. rosea en macetas.
\end{abstract}

Palabras claves: días a floración, longitud de pétalo, área de pétalo, Buenos Aires.

Papone, M.L. and N. Fatta, 2011. Photoperiod effect on commercial quality of Althaea rosea plants obtained from elite germplasm. Agriscientia XXVIII (2): 157-161

\section{SUMMARY}

Althaea rosea has little spread in Buenos Aires despite of its decorative value. Starting from seeds of wild plants and after 3 cycles of selection, we have obtained germplasm with good behavior in pots. With the aim of disseminating A. rosea, we faced tests in pots, with the objective of estimating photoperiod effects on commercial quality of the elite germplasm obtained. The plants that received $16 \mathrm{~h}$ of light showed less number of days until the appearance of the first bud than those receiving natural light. This result is interesting because it 
indicates that the first ones could arrive early for sale. Differences were also found for petal length and area in plants with $16 \mathrm{~h}$ photoperiod, its showed larger flowers and wider petals than tended to overlap when they were compared with those observed in the flowers of plants that received natural light. The plans treated with long photoperiod exhibited less variation coefficient for the measured values, allowing the conclusion that light supplementation is an effective tool to focus the sale and increase quality of $A$. rosea potted plants.

Keywords: days to flowering, petal length, petal area, Buenos Aires.

M.L. Papone. Instituto de Floricultura CIRN INTA-Castelar. De los Reseros y N. Repetto s/n. Villa Udaondo. Buenos Aires. Argentina. N. Fatta. Cátedra de Genética. Facultad de Agronomía. Universidad de Buenos Aires. Argentina. Correspondencia a N. Fatta: nfatta@agro.uba.ar

A. rosea (malva real) es una planta de día largo que, a pesar de ser muy decorativa y de fácil cultivo, tiene escasa difusión en la ciudad de Buenos Aires. Con las finalidades de difundirla en la zona y de obtener resultados que podrían ser de aplicación directa por parte de los floricultores, se practicaron tres ciclos de selección que permitieron obtener germoplasma elite, con buen comportamiento en macetas en Buenos Aires (Papone et al., 2005, 2006, 2007). A continuación se planteó que sería de interés caracterizar la respuesta a la prolongación de las horas de luz, de la población de referencia, con la finalidad de diseñar un protocolo cultural conducente a la obtención de plantas atractivas en maceta.

Se sabe que el fotoperíodo afecta la floración de Arabidopsis thaliana (Koornneef et al., 1998; Cremer \& Coupland, 2003), de gramíneas (Laurie et al., 1994; Castaneda Chavez et al., 2006; Carver, 2009), de leguminosas (Cameron \& Mannetje, 1977; Del Pozo et al., 2000), de hortícolas ornamentales (Mora Cardador, 1997), y comestibles (González et al., 2004) y de otras especies de interés comercial (Bellido, 2002; Varlverde Valdes et al., 2005; Garcia Breijo et al., 2006; Campbell y Reece, 2007). Además, que esta respuesta está genéticamente controlada y que muestra variabilidad (Laurie et al., 1995; Yanovsky \& Kay, 2002; Hayarna et al., 2002; Turner et al., 2005; Carver, 2009; Kilian et al., 2009; Coles et al., 2010). Se hipotetizó, entonces, que prolongar la duración del día con luz artificial por encima de la duración crítica para una gran cantidad de especies de día largo (Curtis \& Barnes, 1997), afectaría la calidad comercial de plantas de A. rosea.

Por tal motivo se diseñó el presente experimento cuyo objetivo fue estimar el efecto de la aplicación de un fotoperíodo considerado largo, de 16 h, sobre caracteres de producción determinantes de la calidad comercial y del valor final en el germoplasma elite obtenido de Althaea rosea.

Durante el invierno 2007 se mantuvieron en macetas de $3 \mathrm{I}$, con un sustrato compuesto por $50 \%$ pinocha, $25 \%$ resaca de río y $25 \%$ tierra bajo invernáculo, plantas de A. rosea logradas en 2006 a partir de semilla elite propia (Papone et al., 2005, 2006). Se regaron diariamente con agua de red y se fertilizaron una vez por semana con: $1 \mathrm{~g} \mathrm{l}^{-1} \mathrm{de}$ $\mathrm{KNO}_{3}, 1 \mathrm{~g} \mathrm{l}^{-1}$ de $\mathrm{NH}_{4} \mathrm{H}_{2} \mathrm{PO}_{4}$ y $0,75 \mathrm{gll}^{-1}$ de $\mathrm{NH}_{4} \mathrm{NO}_{3}$. El 23/7/2007 se eligieron al azar 12 plantas y 6 de ellas se distribuyeron sobre una mesada de rejilla metálica de 2,70 $\mathrm{m}^{2}(3 \mathrm{~m} \times 0,90 \mathrm{~m})$ que contaba con un luxómetro marca $N \& T$, instalado en el centro de la mesada. Todo se instaló en una cámara, la que se reguló con un fotoperíodo de $16 \mathrm{~h}$ de luz diarias con lámparas incandescentes de $100 \mathrm{~W}$, equivalentes a 1,36 micromoles $\mathrm{m}^{-2} \mathrm{~s}^{-1}$. Este conjunto, ubicado en el interior de un invernáculo de polietileno, se aisló del exterior mediante cortinas de material plástico de color negro por dentro y aluminizado por fuera. El resto de las plantas se mantuvo dentro del invernáculo bajo luz natural (Figura 1). El ensayo se extendió durante 7 meses, hasta fines de febrero, cuando se consideró que ya no se produciría diferenciación de flores.

Se contó con un calefactor de encendido automático, para los momentos en que la temperatura descendía por debajo de $10{ }^{\circ} \mathrm{C}$. Por otra parte, el aumento de la temperatura se controló con la apertura de puertas y ventanas. Cada planta se consideró una repetición y se las dispuso al azar en cada ambiente. Se determinó el número de días desde el 23/7/2007 a la aparición del primer pimpollo (DAP) y de la primera flor (DAF) de cada planta. También se midieron la altura de la planta en el día de aparición de la primera flor y el área 
y la longitud de los pétalos de la primera flor, con el programa ImageJ 1,3, y se calculó el cociente entre los dos últimos caracteres.

Los datos se analizaron estadísticamente con análisis de varianza (ANOVA) y las medias se compararon con el test de Tukey, utilizando el programa Infostat/P $\vee 1.1$

No se hallaron diferencias significativas entre tratamientos para altura de la planta ni para DAF $(p=0,32$ y 0,55 , respectivamente). Para DAP se halló una diferencia estadística con un nivel de significancia de $p=0,10$. Las plantas que crecieron con fotoperíodo de $16 \mathrm{~h}$ mostraron un DAP de 32,5 días, y de 47,7 días para el tratamiento de luz natural.

El coeficiente de variación (CV) de DAP fue comparativamente menor para el tratamiento con

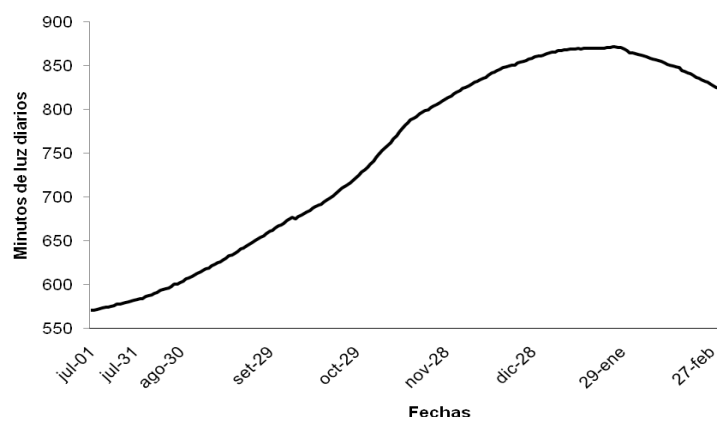

Figura 1. Duración del fotoperíodo en Buenos Aires (34 35"LS, $\left.59^{\circ} 29^{\prime \prime} \mathrm{LO}\right)$ durante el período del ensayo. Fuente The weather channel (http://www espanol.weather.com)

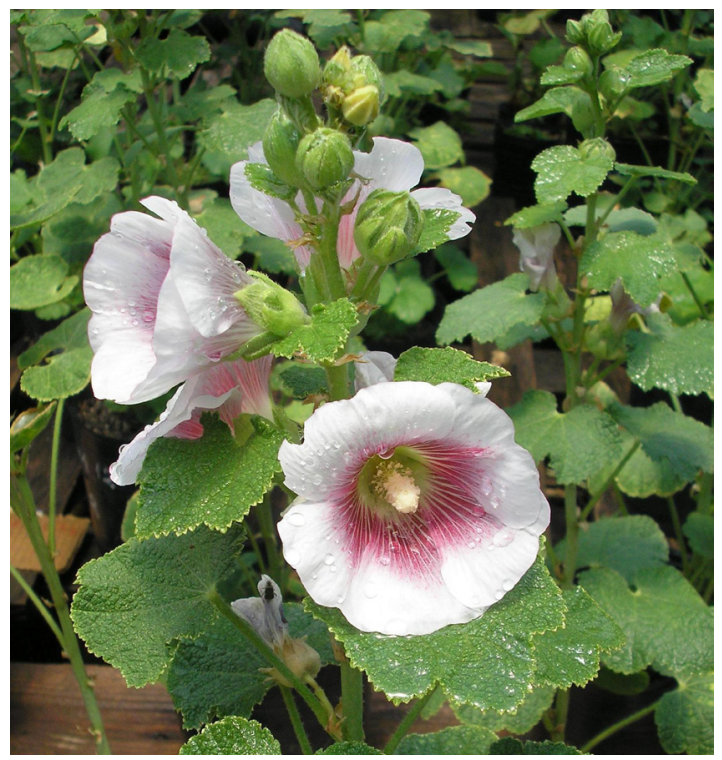

Figura 2. Corola de una de las plantas de Althaea rosea tratada con $16 \mathrm{~h}$ de luz.
16 h de fotoperíodo (Tabla 1), lo que sugiere que con esta práctica cultural el productor podría beneficiarse económicamente concentrando sus envíos para la venta.

Para el área de pétalo se hallaron diferencias significativas entre tratamientos $(p=0,00)$; así, el área de pétalo fue tres veces más grande en las plantas con $16 \mathrm{~h}$ de fotoperíodo con respecto al área de pétalo para las plantas con luz natural (Figuras 2 y 3 ).

También se hallaron diferencias estadísticas para la longitud de los pétalos $(p=0,01)$, que fue mayor con fotoperíodo largo que con luz natural (3,24 cm y 2,73 cm respectivamente). Para el cociente entre las áreas y las longitudes de los pétalos se hallaron diferencias significativas entre tratamientos $(p=0,00)$. Los valores promedio fueron 1,68 y $0,61 \mathrm{~cm}^{2} \mathrm{~cm}^{-1}$, con fotoperíodos largo y natural, respectivamente. Estos últimos resultados ponen en evidencia el beneficio del tratamiento sobre el valor estético de la flor, que es un carácter importante para definir calidad y precio de mercado (Figura 4).

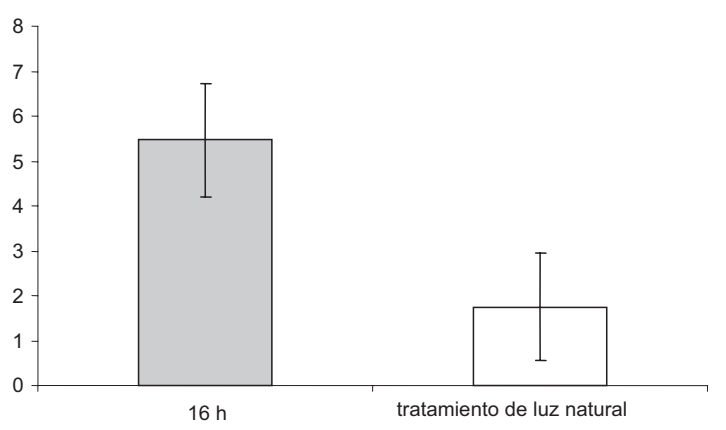

Figura 3. Área de pétalo de la primera flor de cada planta de Althaea rosea para fotoperíodos de $16 \mathrm{~h}$ y natural.

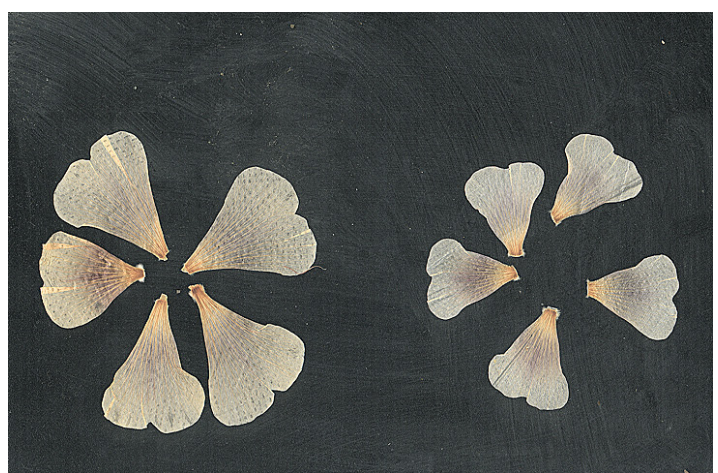

Figura 4. Imágenes de scanner de pétalos de plantas de Althaea rosea con $16 \mathrm{~h}$ de fotoperíodo (izquierda) y con luz natural (derecha) 
Los CV del área y de la longitud de los pétalos y del cociente entre ambos, fueron mayores para el tratamiento de luz natural (Tabla 1).

Tabla 1. CV para DAP, para área y longitud de pétalo y para el cociente área $x$ longitud de pétalo ${ }^{-1}$

\begin{tabular}{lcccc}
\hline & $\begin{array}{c}\text { DAP } \\
\text { (días) }\end{array}$ & $\begin{array}{c}\text { Area de } \\
\text { pétalo }\end{array}$ & $\begin{array}{c}\text { Longitud de } \\
\text { pétalo }\end{array}$ & $\begin{array}{c}\text { Relación área/ } \\
\text { longitud de pétalo }\end{array}$ \\
\hline $\begin{array}{l}16 \text { h de } \\
\text { luz }\end{array}$ & 0,31 & 0,23 & 0,10 & 0,21 \\
$\begin{array}{l}\text { Luz } \\
\text { natural }\end{array}$ & 0,37 & 0,69 & 0,31 & 0,52 \\
\hline
\end{tabular}

La diferencia para DAP indica que las plantas que recibieron $16 \mathrm{~h}$ de luz podrían llegar a un estado apropiado para la venta antes que las obtenidas con luz natural, lo que constituye un resultado comercialmente interesante. Además, estas plantas mostraron flores atractivas no sólo por su mayor tamaño y por sus pétalos más anchos, sino también porque los pétalos tendieron a solaparse (Figura 2) y originaron corolas visualmente más atractivas.

Varios caracteres de interés comercial, fueron profundamente afectados por el tratamiento, y estos resultados coinciden con las observaciones de Thomas (1998), Dunlap (1999) y Kolosova et al. (2001). Estos autores consignan un gran número de caracteres que muestran oscilaciones periódicas explicadas en parte por factores ambientales, entre ellos las horas de luz.

Los menores valores de los CV en las plantas que se mantuvieron con luz controlada resultan de aplicación práctica, pues la coincidencia de los individuos en sus estados fenológicos simplifica la actividad empresarial y permite concentrar la operación de envío del producto al mercado.

La prolongación del fotoperíodo con luz artificial resultó una herramienta efectiva para aumentar la calidad de las plantas en macetas en el momento en que estén en condiciones de ser destinadas a la venta.

Sería de interés hacer nuevas determinaciones con un número mayor de repeticiones. Sin embargo, los resultados son promisorios y contribuyen a la caracterización de la población de referencia, la cual podría ser el punto de partida de una variedad vegetal de $A$. rosea de flor para maceta.

\section{AGRADECIMIENTOS}

Al Ing. Agr. Diego Mata y al Ing. Agr. Juan Carlos Hagiwara por el asesoramiento en el uso de las instalaciones para fotoperíodo con luz artificial.

\section{BIBLIOGRAFIA}

Bellido, L.L., 2002. Cultivos Industriales. Ediciones Mundi Prensa. España. pp. 793-794.

Cameron, D.F. and L.T. Mannetje, 1977. Effects of photoperiod and temperature on flowering of twelve Stylosanthes especies. Australian Journal of Experimental Agriculture and Animal Husbandy. 17: 417-424.

Campbell, N.A. and J.B. Reece, 2007. Biología. $7^{a}$ edición. Editorial Médica Panamericana S.A. España. pp. 806.

Castaneda Chavez, J. W.; S.C. Mason, Q. Argueta PortiIlo, E. R. Ventura, M. Hernández Valle y R.C. Valencia, 2006. Eficiencia del uso de Nitrógeno en sorgo sensible al fotoperíodo en asocio con maíz en El Salvador. Agronomía Mesoamericana. 17:1 11-17.

Carver, B.F., 2009. Wheat science and Trade. Wiley Blackwell (eds). USA. pp. 58-60.

Coles, N.D.; M.D. McMullen, P.J. Balint-Kurti, R.C. Pratt and J.B. Holland, 2010. Genetic Control of Photoperiod Sensitivity in Maize Revealed by Joint Multiple Population Análisis. Genetics 184: 799-812.

Cremer, F. and G. Coupland, 2003. Distinct photoperiodic responses are conferred by the same genetic pathwat in Arabidopsis and in rice. Trends in Plant Science 8:9 405-407.

Curtis, H. and N.S. Barnes, 1997. Invitación a la Biología. $5^{\circ}$ ed. Editorial Médica Panamericana. España. pp. 764-768.

Del Pozo, A.; C. Ovalle, J. Aronson and J. Avendaño, 2000. Developmental responses to temperature and photoperiod in ecotypes of Medicago polymorpha L. along an environmental gradient in Central Chile. Annals of Botany. 85:6 809-814.

Dunlap, J.C., 1999. Molecular bases for circadian clocks. Cell 96 2: 271-290.

The weather channel (http://www espanol.weather.com, consultado el 18/09/2004)

García Breijo, F.J.; J. Rosello Caselles and M.P. Santamarina Siurana, 2006. Introducción al funcionamiento de las plantas. Editorial Universidad politécnica de Valencia. España. pp.121-126.

González, M.I.; A. del Pozo; D. Cotroneo and R. Pertierra, 2004. Días a floración en espinaca (Spinacia oleracea L.) en diversas épocas de siembra: respuesta a la temperatura y al fotoperíodo Agric. Téc. (on line) 64 (4) 331-337. ISSN 0365-2807. doi: 10.4067/S036528072004000400001.

Hayarna, R.; T. Izawa and K. Shimamoto, 2002. Isolation of rice genes possibly envolved in the photoperiodic control of flowering by fluorescent differential display method. Plant Cell Physiol. 43 5: 494-504. 
ImageJ Image processing and Análisis in Java (http:// www.rsbweb.nih.gov/ij, consultado el 12/03/2004).

InfoStat Software Estadístico (http://www.infostat.com.ar/ index.php, consultado el 08/12/2004).

Kilian, B.; H. Ozkan; C. Pozzi and F. Salamini, 2009. Domestication of Triticeae in the fertile Crescent In: Genetics and Genomics of the Triticeae. Vol. 7. Feuillet C., Muehlbauer G. J. (eds). USA. Cap 3 p. 81-21.

Kolosova, N.; N. Gorenstein; C. Kisch and N. Dudareva, 2001. Regulation circadian methyl benzoate emission in diurnally and nocturnally emitting plants. The Plant Cell 13: 2333-2347.

Koornneef, M. C.; A.J. Alonso-Blanco; M. Peeters and W. Soppe, 1998. Genetic control of flowering time in Arabidopsis. Annual Review in Plant Physiology and Plant Molecular Biology 49: 345-370

Laurie, D.A.; N. Pratchett; J.W. Snape; J.H. Bezant and J.W. Snape, 1994. Genetic analysis of a photoperiod response gene on the short arm of chromosome $2(2 \mathrm{H})$ of Hordeum vulgare (barley). Heredity 72: 619.627.

Laurie, D.A.; N. Pratchett; J.W. Snape and J.H. Bezant, 1995. RFLP mapping of five major genes and eight quantitative trait loci controlling flowering time in a winter x spring barley (Hordeum vulgare L.) cross. Genome. 38: 575-585.

Mora Cardador, L., 1997. Respuesta de 5 variedades de crisantemo (Dendrathema grandiflora Tzevel) al manejo de fotoperíodo bajo cubierta plástica oscura en Nezahuacoyotl, Texcoco, Estado de México. http:// agris.fao.org/agrissearch/ search/display.

Papone, M.L.; D. Tripicchio; A. Mascarini y N.A. Fatta, 2005. Evaluación del comportamiento en Buenos Ai- res de una población rionegrina de Althaea rosea. Libro de Resúmenes formato CD. VII Jornadas Nacionales de Floricultura. Chubut. Argentina. http://alepo. agro.uba.ar/publicaciones/FAUBAenRevistas.pdf

Papone, M.L.; D. Tripicchio; A. Mascarini y N.A. Fatta, 2006. Comportamiento de la descendencia de plantas cultivadas y seleccionadas en Buenos Aires a partir de una población rionegrina de Althaea rosea. Libro de Resúmenes III Congreso Argentino de Floricultura y VIII Jornadas Nacionales de Floricultura. Buenos Aires. Argentina. p. 81-84. http://alepo.agro.uba.ar/ publicaciones/FAUBAen Revistas.pdf

Papone, M.L.; A. Mascarini y N.A. Fatta, 2007. Mejoramiento genético en Althaea rosea; $3^{\text {er }}$ ciclo de selección. Libro de Resúmenes IX Jornadas Nacionales de Floricultura. 14-16/11/2007. Salta. Argentina. pp. 211213.

Thomas, B., 1998. Photoperiodism: an overview. In: Biological rhythms and photoperiodism in plants. Lumsden P.J., Millar A.J. Publishers Bios Scientific Publishers U.K. pp. 151-160.

Turner, A.; J. Beales; S. Faure; R.P. Dunford and D.A. Laurie, 2005. The pseudo-response regulator Ppd-H1 provides adaptation to photoperiod in barley. Science 310: 1031-1034.

Varlverde Valdes, T.; J.A. Meave del Castillo; J. Carabias Lillo y Z. Cano Santana, 2005. Ecología y medio ambiente. Pearson Educación de México S.A. de C.V. México. pp. 27.

Yanovsky, M.J. and S.A. Kay, 2002. Molecular basis of seasonal time measurement in Arabidopsis. Nature 419: 308. 